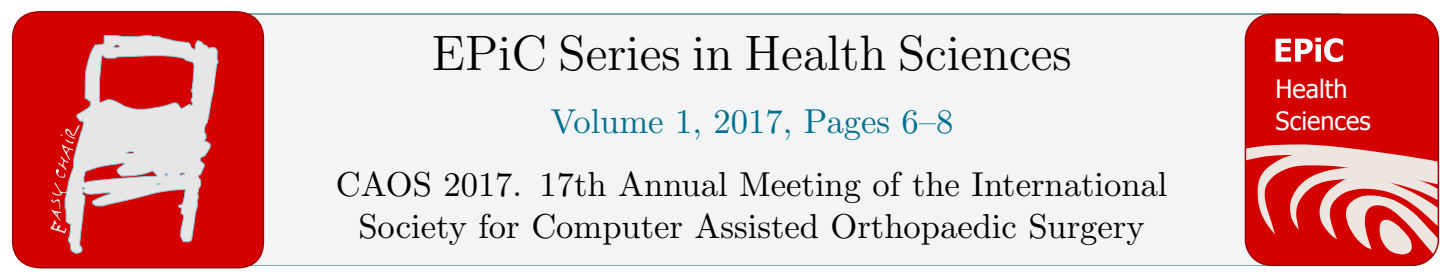

\title{
Ten year survival of navigation-assisted total knee arthroplasty
}

\author{
Jean-Yves Jenny \\ University Hospital Strasbourg, Strasbourg, France \\ jean-yves.jenny@chru-strasbourg.fr
}

\begin{abstract}
The survival rate of navigation-assisted total knee arthroplasty when mechanical revision was considered as the end-point was $98 \%$ after 10 years and $96 \%$ after 15 years. These figures compares favorably with previously published literature about conventional implantation. Longer follow-up is required to prove superiority of any technique.
\end{abstract}

\section{Introduction}

Total knee arthroplasty (TKA) is considered a highly successful procedure. Survival rates of more than $90 \%$ after 10 years are generally reported. However, complications and revisions may still occur for many reasons, and some of them may be related to the operative technique. Computer assistance has been suggested to improve the accuracy of implantation of a TKA (Jenny 2005). Short term results are still controversial (Roberts 2015). However, few long term results have been documented (Song 2016). The present study was designed to evaluate the long-term (more than 10 years) results of a TKA which was routinely implanted with help of a non-image based navigation system. The 5- to 8year of this specific TKA has already been documented (Jenny 2013).

The hypothesis of this study will be that the 10 year survival rate of this TKA will be improved in comparison to historical papers when analyzing survival rates and knee function as evaluated by the Knee Society Score (KSS).

\section{Material and methods}

All patients operated on between 2001 and 2004 for implantation of a navigated TKA were eligible for this study. Usual demographic and peri-operative items have been record. All patients were prospectively followed with clinical and radiological examination. All patients were contacted after the 10 year follow-up for repeat clinical and radiological examination (KSS, Oxford knee 
questionnaire and knee plain X-rays). Patients who did not return were interviewed by phone call. For patients lost of follow-up, family or general practitioner was contacted to obtain relevant information about prosthesis survival. Survival curve was plotted according to Kaplan-Meier.

\section{Results}

247 TKAs were implanted during the study time-frame. 225 cases had an optimal lower limb axis (HKA angle between $177^{\circ}$ and $183^{\circ}$ ) after TKA (91\%). Final follow-up (including death or revision) was obtained for 200 cases (81\%). Clinical status after 10 years was obtained for 146 cases (59\%) (KSS, 102 cases - Oxford questionnaire, 146 cases - radiologic evaluation, 94 cases). 4 prosthetic revisions were performed for mechanical reasons during the follow-up time (1\%). The 10 year survival rate was $98 \%$. The mean KSS was 188 points. The mean Oxford score was 55 points. No component was considered loose at the final radiographic evaluation. No polyethylene wear was detected at the final radiographic evaluation.

\section{Discussion}

This study confirms our initial hypothesis, namely quite satisfactory results of navigated implanted TKA after more than 10 years. Navigation, whose precision is no longer to be demonstrated, probably contributed to the quality of the results. A more consistent anatomical reconstruction and ligamentous balance of the knee should lead to more consistent survival of the TKA. Other authors did observe similar results (Baumbach 2016). However, superiority of navigated TKA in comparison to conventional implanted TKA is difficult to prove because of the subtle differences expected in mostly underpowered studies. Longer term follow-up may be required.

\section{References}

1. Baumbach JA, Willburger R, Haaker R, Dittrich M, Kohler S, 10-year survival of navigated versus conventional TKAs: a retrospective study, Orthopedics, 39(3 Suppl), pp: S72-76, 2016.

2. Jenny JY, Miehlke R, Saragaglia D, Geyer R, Mercier N, Schoenahl JY, Thiel B, Single-radius, multidirectional total knee replacement, Knee Surg Sports Traumatol Arthrosc, 21, pp: 2764-2769, 2013.

3. Jenny JY, Clemens U, Kohler S, Kiefer H, Konermann W, Miehlke RK, Consistency of implantation of a total knee arthroplasty with a non-image-based navigation system: a case-control study of 235 cases compared with 235 conventionally implanted prostheses, J Arthroplasty, 20(7), pp: 832-839, 2005.

4. Roberts TD, Clatworthy MG, Frampton CM, Young SW, Does computer assisted navigation improve functional outcomes and implant survivability after total knee arthroplasty?, J Arthroplasty, 30(9 Suppl), pp: 59-63, 2015.

5. Song EK, Agrawal PR, Kim SK, Seo HY, Seon JK, A randomized controlled clinical and radiological trial about outcomes of navigation-assisted TKA compared to conventional TKA: long-term follow-up, Knee Surg Sports Traumatol Arthrosc, 24(11), pp: 3381-3386, 2016. 


\section{Disclosure}

Jean-Yves JENNY receives royalties from Aesculap, is a paid consultant for Exactech, is an unpaid consultant for FH Orthopedics, is member of the board of the CAOS-International Society and of the French Society for Hip and Knee Surgery 